\title{
Comparing The Efficacy Of An Anti-Human VEGF-A Neutralizing Antibody Versus Bevacizumab On A Laser-Induced Choroidal Neovascularization (CNV) Rhesus Monkey Model
}

This article was published in the following Dove Press journal:

Drug Design, Development and Therapy

\author{
Oscar Olvera-Montaño (D) \\ Leopoldo Baiza-Duran' \\ Juan D Quintana-Hau ${ }^{2}$ \\ Mayra G \\ Quiñonez-Alvarado \\ Wen Zeng ${ }^{3}$ \\ Li Gong ${ }^{3}$ \\ Patricia Muñoz-Villegas (D) \\ 'Clinical Research Department, \\ Laboratorios Sophia, SA De CV, \\ Zapopan, Jalisco, Mexico; ${ }^{2}$ Research and \\ Development Department (CIS), \\ Zapopan, Jalisco, Mexico; ${ }^{3}$ Sichuan \\ Primed Shines Bio-Tech Co, Ltd, \\ Chengdu, Sichuan, People's Republic of \\ China
}

Purpose: To evaluate the efficacy of a therapy on improving characteristics of laser-induced choroidal neovascularization (CNV) via single intravitreal injection of a humanized antihuman VEGF monoclonal antibody (PRO-169) versus bevacizumab in a rhesus monkey model.

Methods: To induce experimental CNV, small high-energy laser spots were used to treat several areas, around the macula in the retinas of monkeys at Day -21 . Eighteen rhesus monkeys were used for CNV induction. The efficacy endpoints were fluorescein leakage by FFA and retinal thickness by OCT. FFA examinations were performed 19 days after induction. Appropriate animals were enrolled for treatment and randomly divided into 3 groups: bevacizumab ( $\mathrm{n}=5,7$ eyes), PRO-169 ( $\mathrm{n}=5,7$ eyes), and vehicle controls ( $\mathrm{n}=4,7$ eyes).

Results: In 25 of 36 (69.4\%) eyes, CNV lesions were identified. The average percent change of retinal thickness in the eyes of bevacizumab group was $-159.3 \pm 62.2 \%$ and $-154.0 \pm 45.1 \%$ $(p<0.01$ vs Vehicle) at Day 14 and Day 28, respectively; in the eyes of PRO-169 group it was $-131.6 \pm 68.7 \%$ and $-131.5 \pm 63.8 \%$ ( $p<0.01$ vs Vehicle), respectively. The average percent change of leakage area in the eyes of bevacizumab group was $-75.3 \pm 49.4 \%$ and $-78.0 \pm 42.6 \%$ ( $p<0.01$ vs Vehicle) at Day 14 and Day 28, respectively; in the eyes of PRO-169 group it was $-82.0 \pm 19.3 \%$ and $-81.4 \pm 21.0 \%$ ( $p<0.01$ vs Vehicle), respectively. There were no abnormalities found in behavior, skin and hair, excretion and overall eye appearance before and after treatment in all groups.

Conclusion: After photocoagulation, the eyes enrolled in this studio showed CNV related characteristics including increased retinal thickness, and fluorescein leakage at laser spots. PRO-169 (1.25 mg per eye) can reduce the retinal thickness and fluorescein leakage area after treatment for 14 and 28 days in this rhesus monkeys model, without toxic effect or adverse events. These findings suggested that PRO-169 can inhibit CNV.

Keywords: age-related macular degeneration, monoclonal antibodies, fundus fluorescein angiography, optical coherence tomography

\section{Introduction}

Choroidal neovascularization (CNV) comprises the invasion of new blood vessels from the choroid through breaks in Bruch's membrane into the subretinal pigment epithelial and subretinal space. ${ }^{1,2}$ The CNV leads to the severely progressive decrease of visual acuity (VA) of neovascular/wet age-related macular degeneration (AMD). ${ }^{3,4}$ AMD is a highly complex disease that affects millions of people
Laboratorios Sophia, SA de CV, Paseo de Norte 5255, Guadalajara Technology Park, Zapopan 45010 , Jalisco, Mexico Tel +52 330l 4200, Ext: 1018 Fax +5233014200

Email patricia.munoz@sophia.com.mx 
worldwide and is a leading cause of irreversible blindness among the elderly in developed countries. ${ }^{5,6}$ Over the past 20 years, it has become evident that antibody-based inhibitors of vascular endothelial growth factor (VEGF) present effective treatment for AMD, leading to clinically significant improvement of VA in a substantial number of patients.

Ocular neovascularization provides an ideal in vivo system in which to investigate the mechanism that control angiogenesis. ${ }^{7}$ There are several antiangiogenic molecules that target VEGF (also called VEGF-A) and are used in the management of AMD. ${ }^{8-10}$ Monoclonal antibodies (mAbs) can be used therapeutically in ophthalmology by binding to molecular targets with high specificity. ${ }^{11}$ Monoclonal antibodies inhibiting VEGF signaling (ranibizumab, bevacizumab) have provided to be effective in neovascular ocular disorders like AMD ${ }^{1,2,12-14}$ Ranibizumab is an anti-VEGF antibody fragment, specifically designed for ophthalmic use, was developed for intraocular administration and born from the possibility that side effects might occur in an elderly population, such as those with AMD, and from toxicity in clinical trials with systemic administration of bevacizumab. ${ }^{15}$

PRO-169 is a humanized-anti-VEGF mAb with a molecular mass of $149 \mathrm{kDa}$. PRO-169 had a target specificity similar to bevacizumab (Avastin, Genentech). ${ }^{9,14}$ Bevacizumab is a mAb approved by the United States Food and Drugs Administration for cancer therapy and used off-label to treat neovascular AMD and other ocular disease associated with macular edema and abnormal vessel growth. ${ }^{10,15}$ In head-to-head comparisons of ranibizumab with bevacizumab, the drugs had equivalent effects on VA throughout the first year of follow-up. ${ }^{13,14}$

Recent studies evaluating the efficacy of bevacizumab and ranibizumab in rodent models of laser-induced $\mathrm{CNV},{ }^{10,16,17}$ demonstrated that these mAbs anti-VEGF offer no improvement in leakage or $\mathrm{CNV}$ in rat and mice. This absence of efficacy is supposed to be related to the structural differences between rodent and human VEGF (species-specific). ${ }^{1,16,18-20}$ Bevacizumab is humanspecific and does not react with rat VEGF because of an amino acid substitution in the bevacizumab-binding site. ${ }^{20}$ Thus, the inhibition of corneal neovascularization of $\mathrm{mAb}$ is far from complete in murine models. ${ }^{17}$ However, the laser-induced CNV model in non-human primates (NHPs) has played a critical role in the development of new drugs for AMD. ${ }^{21}$ Although antibodies to VEGF have been shown to inhibit neovascularization in an experimental model of laser-induced CNV in rhesus monkeys. ${ }^{3,21}$ This model uses laser, to induce CNV lesions with significant angiographic leakage, ${ }^{1,12}$ and is a reliable and reproducible model that has been widely studied and is well characterized in the literature. ${ }^{1,3,5}$ The rhesus monkey model has relevance for preclinical pharmacologic studies due to the anatomic similitudes between NHPs and human eye, ${ }^{22}$ and has become a model of choice for preclinical evaluation of the efficacy and safety of VEGF inhibitors. ${ }^{1}$

The purpose of this in vivo preclinical study was to assess the efficacy and safety of intravitreal injection of mAb PRO-169 versus bevacizumab on improving characteristics of laser-induced $\mathrm{CNV}$ rhesus monkey model.

\section{Materials And Methods}

\section{Animal Experiments}

All animal studies were following the Animal Welfare Act, the Guide for the Care and Use of Laboratory Animals, and the Office of Laboratory Animal Welfare; they were approved by Institutional care and use committee (IACUC) of Sichuan PriMed Shines Bio-Tech Co., Ltd.

\section{Monoclonal Antibodies And Vehicle}

Positive control: Bevacizumab (Avastin; Genentech, South San Francisco, CA, USA) is a commercially available monoclonal antibody. Test article: PRO-169 is a mAb Anti-VEGF (Laboratorios Sophia, SA de CV, Zapopan, Jalisco, Mexico by KBI Biopharma), and placebo: the vehicle was a sterile, clear solution, $\mathrm{pH}=6.2$.

\section{Induction Of CNV And Drug Delivery}

A total of 18 rhesus monkeys of Chinese origin, obtained from Primed Non-human Primate Research Center (Ya'an, China), were used for CNV induction. The groups conformed are summarized in Table 1. Monkeys weighed $3.5-4.8 \mathrm{~kg}$ and aged from 3 to 5 years ( 9 males and 9 females). Baseline fundus photography (FP), fundus fluorescein angiography (FFA), and optical coherence tomography (OCT) were performed as part of animal and ocular health screening (Day -31). The exclusion criteria were serious inflammation in eye post laser-induction and having no point of grade III or IV of fluorescence leakage in both of the eyes. The CNV technique is based on previously published methods. ${ }^{1,3,12}$ On Day -21 , after animals were anesthetized, both eyes of each monkey were treated with laser at 9 spots around the macula (laser wavelength $532 \mathrm{~nm}$, diameter of facula $50 \mu \mathrm{m}$, energy 600-775 mW, time of exposure $0.1 \mathrm{~s}$ ). The laser (Vitra 532 $\mathrm{nm}$, Quantel Medical) was mounted on a slit lamp with an 
Table I Treatment groups

\begin{tabular}{|c|c|c|c|c|c|c|c|}
\hline Groups $^{a}$ & $\begin{array}{l}\text { Number Of } \\
\text { Animals Enrolled }\end{array}$ & $\begin{array}{l}\text { Number Of Eyes } \\
\text { Enrolled }\end{array}$ & $\begin{array}{l}\text { Dose Conc. } \\
(\mathrm{mg} / \mathrm{mL})\end{array}$ & $\begin{array}{l}\text { Dose } \\
\text { Volume } \\
(\mu \mathrm{L})\end{array}$ & $\begin{array}{l}\text { Dose } \\
\text { Amount } \\
\text { (mg) }\end{array}$ & $\begin{array}{l}\text { Route Of } \\
\text { Dosing }\end{array}$ & Endpoints \\
\hline $\begin{array}{l}\text { Vehicle } \\
\text { Bevacizumab } \\
\text { PRO-169 }\end{array}$ & $\begin{array}{l}4 \\
5 \\
5\end{array}$ & $\begin{array}{l}7 \\
7 \\
7\end{array}$ & $\begin{array}{l}0 \\
25 \\
25\end{array}$ & 50 & $\begin{array}{l}0 \\
1.25 \\
1.25\end{array}$ & IVT & $\begin{array}{l}\text { FP, FFA, } \\
\text { OCT. }\end{array}$ \\
\hline
\end{tabular}

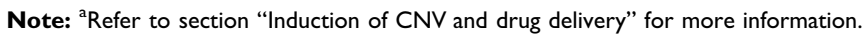

Abbreviations: Ivt, intravitreal injection; FP, fundus photography; FFA, fundus fluorescein angiography; OCT, optical coherence tomography.

adapter and the beam directed to the retina with a Volk Centralis diameter (Volt Optical, Mentor, OH). FFA was performed 19 days later. If there was more than 1 point with fluorescence leakage of grade III or IV lesion, the eye was considered clinically relevant and modeling was assessed as successful. ${ }^{1,3,5}$ According to the area of III+IV grade of fluorescein leakage spots, appropriate animals were enrolled for treatment and randomly divided into three groups: vehicle $(\mathrm{n}=4)$, bevacizumab $(\mathrm{n}=5)$ and PRO-169 $(\mathrm{n}=5)$. Importantly, the initial and only injection was delivered on postoperative Day 21 for all groups. After the animal was anesthetized with intramuscular injection of 1:1 ketamine-xylazine mix $(10 \mathrm{mg} / \mathrm{kg})$, a $5 \%$ povidone iodine solution (Chengdu Yogan Pharmaceutical Co, Ltd.) was placed in the conjunctival sac, eyelid and eyelash for disinfection. A 29-gauge needle attached to a syringe containing test or control article ( $50 \mu \mathrm{L}$ bevacizumab $1.25 \mathrm{mg}$ per eye), PRO$1691.25 \mathrm{mg}$ per eye or vehicle) was used for intravitreal (IVT) injection through an area $2-3 \mathrm{~mm}$ posterior to the limbus, aiming toward the center of globe. A broad-spectrum antibiotic eye drop was applied to the eyes (Oculentum Erythromycin, Xinxiang Huaqing Pharmaceutical Co., Ltd).

\section{Clinical Observation}

Each animal was observed once daily by an examiner who was blinded to the treatment groups to minimize the observer bias. Each monkey was fed with $200 \mathrm{~g}$ diet every day (protein $17 \%$, fat $5 \%$, carbohydrate $63 \%$ ). Food consumption was assessed semi-quantitatively, abnormal findings were recorded. Animals got access to water all the time and 2 monkeys were pair-housed every day during the study. The body weight was weighed once before induction, once before administration, and once at Day 14 and Day 28 .

\section{Fundus Photography (FP)}

FP was conducted once before induction (Day -31); once after induction (Day -21); once before test article administration (Day -2); once 2 weeks after administration (Day 14); and once 4 weeks after administration (Day 28), see Figure 1. Animals were anesthetized with 1:1 ketamine-xylazine mix
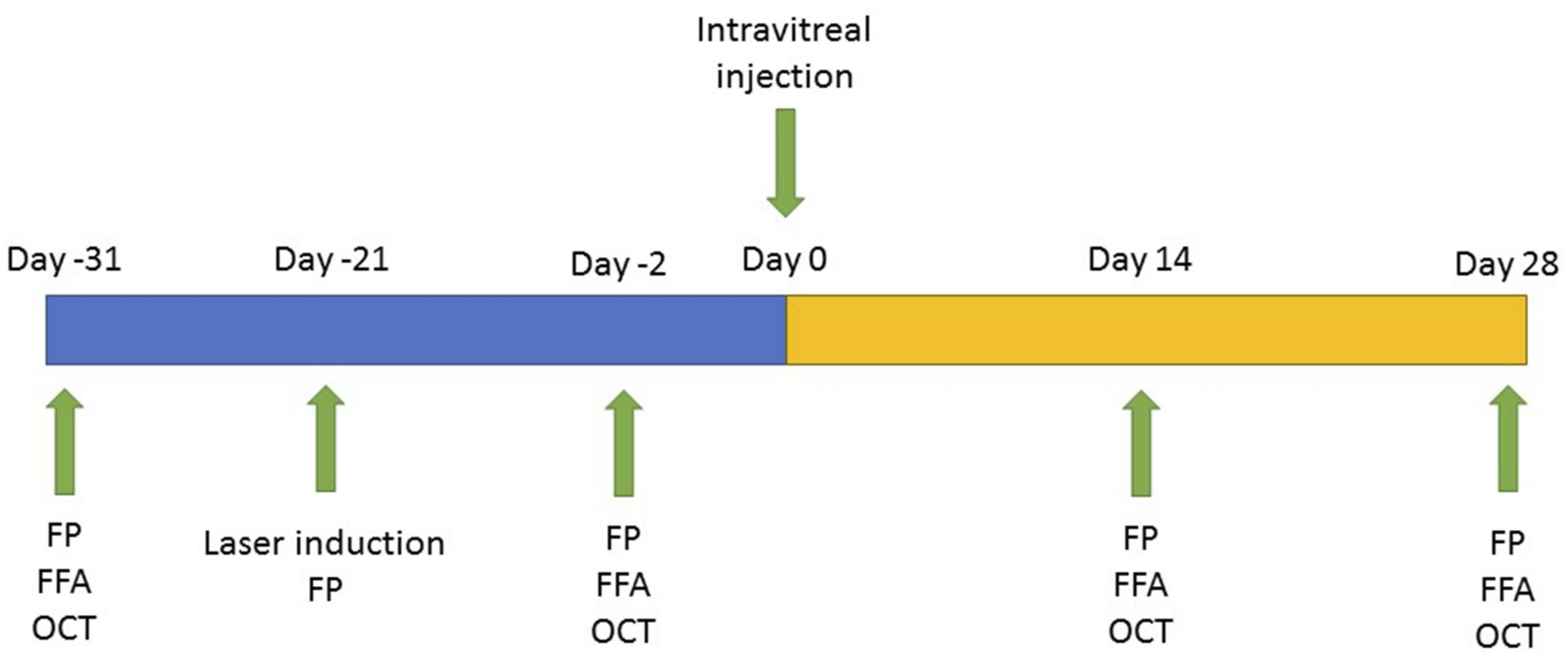

Figure I Study design.

Abbreviations: FP, fundus photography; FFA, fluorescein fundus angiography; OCT, optical coherence tomography. 
(10 mg/Kg), 2 drops of tropicamide phenylephrine eye drop (Santen Pharmaceutical Co., Ltd) were applied to each eye for pupil dilation. The monkeys were placed in a dark room until pupil diameter was greater than $6 \mathrm{~mm}$. FP and angiography were performed using a retinal camera (VX-20, Kowa Company, Ltd).

\section{Outcome Measurement} Retinal Thickness By OCT

The retinal thickness of laser spots was assessed by Optical Coherence Tomography (OCT) once before induction (Day -31 ); once after induction (Day -21 ); once before test article administration (Day -2 ); once 2 weeks after administration (Day 14); and once 4 weeks after administration (Day 28), see Figure 1. OCT (Spectralis OCT Plus, Heidelberg Engineering $\mathrm{GmbH}$ ) images were acquired after FP and FFA. To ensure high-quality OCT images, the eyes were evenly illuminated, and the camera was well focused on the macular fovea. The fast-macular scan procedure was applied to the eyes. The builtin software of Heidelberg OCT was used to measure the retinal thickness by determining the distance between the inner limiting membrane and base membrane BM automatically. Occasionally, manually locating the 2 membrane lines was necessary. The location with the maximal thickness around the burn spot was chosen for measurement. At 14 and 28 days after dosing, the same OCT examination was applied. Tracking mode was applied to keep the analysis consistent.

\section{Amount Of Grade III And IV Spots By FFA}

Fluorescein angiography was performed once before induction (Day -31); once after induction (Day -21); once before test article administration (Day -2 ); once 2 weeks after administration (Day 14); and once 4 weeks after administration (Day 28), see Figure 1. Rapid series of photographs were taken every $3 \mathrm{~s}$ during the first $1 \mathrm{~min}$ after fluorescein (Alcon Laboratories, Inc) injection to ensure that enough pictures centered on macular fovea at the early stage were collected for both eyes. 5 and 10 mins after fluorescein injection, two photographs were taken for both eyes centered on macular fovea. CNV was rated on an I to IV scale, where $\mathrm{I}=$ no hyperfluorescence; II= lesions exhibited hyper fluorescence early or mid-transit with late leakage; III= lesions showed hyperfluorescence without leakage; IV= lesions showed bright hyperfluorescence early or mild-transit with late leakage extending beyond the borders of the burned area. Lesions were graded systematically from the 12 o'clock position in a clockwise manner. The grade III and IV of fluorescein leakage spots were set as ROIs (region of interest). ${ }^{3}$ The area of
ROI was measured by tracing the borders of fluorescence leakage using Image J software (Wayne Rasband, Research Service Branch, NIH, Bethesda, Maryland, US) with a fixed calibration.

The data that support the findings of this study are openly available in Open Science Framework (https://osf.io) at DOI 10.17605/OSF.IO/NA63J.

\section{Statistical Analyses}

Statistical analyses were carried out using SPSS 19.0 software for Windows (SPSS Inc., Chicago, IL, USA). Microsoft ${ }^{\circledR}$ Office Excel 2016 was used for data processing. Data for analysis consist of animal weights, the area of fluorescein leakage, amount of fluorescein leakage spots, and retinal thickness. The data of percentage change of area of fluorescein leakage spot, percentage change of retinal thickness and change of body weight were analyzed by one-way ANOVA. Data are expressed as means \pm standard deviation (SD). The Tukey's HDS test was applied for multiple comparisons. The amount of fluorescein leakage spots was analyzed using Chisquare test. All statistical analyses performed in this study were with $P$ values $\leq 0.05$ considered statistically significant.

\section{Results}

\section{Retinal Thickness}

A highly reflective light echogenic mass was found in monkey's eyes 19 days after laser photocoagulation, which represented the formation of CNV (red arrows in Figure 2). The retinal thickness of laser spots increased significantly. The percent change of retinal thickness of the laser spot with the maximum retinal thickness was calculated as a sign of efficacy. In the eyes of vehicle group, the average percent change of retinal thickness was $-13.4 \pm 46.4 \%$ and $-5.7 \pm 76.2 \%$ at Day 14 and Day 28, respectively, see Table 2. In the bevacizumab group (1.25 $\mathrm{mg}$ per eye), the inhibition of CNV was found (blue arrows in Figure 2). The average percent change of retinal thickness was $-159.3 \pm 62.2 \%$ and $154.0 \pm 45.1 \%$ at Day 14 and Day 28, respectively (see Table 2). Compared with vehicle, the group treated with bevacizumab had a significant decrease on the retinal thickness $(p=0.001)$. Meanwhile, in the eyes of PRO-169 group (1.25 mg per eye), the inhibition of $\mathrm{CNV}$ was found (blue arrows in Figure 2). The average percent change of retinal thickness was $-131.6 \pm 68.7 \%$ and $-131.5 \pm 63.8 \%$ at Day 14 and Day 28 , respectively, see Table 2 . Compared with vehicle, PRO169 decreased the retinal thickness significantly $(p=0.004)$. No significant differences were observed between 




Figure 2 Representative optical coherence tomography (OCT) images for vehicle, bevacizumab and PRO-169 on pre-laser induction, pre-treatment, Days I4 and 28 after intravitreal injection. The high reflect light echogenic mass (red arrows) was detected in the eyes on Day 19 after laser photocoagulation (Day -2 ). The light echogenic mass decreases after IVT injection (Day I4 and 28) compared with pre-treatment in bevacizumab and PRO-I69 groups (blue arrows). 
Table 2 Change Of Retinal Thickness Of Lesion Spot (\%)

\begin{tabular}{|l|l|l|l|l|}
\hline & $\begin{array}{l}\text { Vehicle, } \\
\mathbf{n = 7} \text { Eyes }\end{array}$ & $\begin{array}{l}\text { Bevacizumab, } \\
\mathbf{n = 7} \text { Eyes }\end{array}$ & $\begin{array}{l}\text { PRO-169, n=7 } \\
\text { Eyes }\end{array}$ & $\mathbf{p}$ \\
\hline Day 14 & $-13.4 \pm 46.4$ & $-159.3 \pm 62.2^{* *}$ & $-131.6 \pm 68.7^{* *}$ & 0.001 \\
Day 28 & $-5.7 \pm 76.2$ & $-154.0 \pm 45.1^{* *}$ & $-131.5 \pm 63.8^{* *}$ & 0.001 \\
\hline
\end{tabular}

Notes: Data are presented as \% mean \pm SD. One-way ANOVA, **compared with vehicle group.

bevacizumab and PRO-169 groups at any time point ( $p=0.668$ and $p=0.784$, respectively).

\section{Leakage Area Of Grade III And Grade IV Spots}

Fluorescein leakage was found in monkey's eyes 19 days after photocoagulation (pre-treatment), which represented the formation of $\mathrm{CNV}$ (red arrows in Figure 3). The average percent change of leakage area of grade III and IV spots was calculated as a sign of efficacy. In the eyes of vehicle, the leakage area slightly decreases caused by selfhealing at Day 14 and Day 28. The average percent change of leakage area \pm standard deviation was $-27.6 \pm 35.3 \%$ and $-36.1 \pm 34.4 \%$ at Day 14 and Day 28, respectively, see Table 3. In the eyes of bevacizumab group, the leakage area reduced significantly (blue arrows in Figure 3). The average percent change of leakage area was $-75.3 \pm 49.4 \%$ and $78.0 \pm 42.6 \%$ at Day 14 and Day 28, respectively, see Table 3. Compared with vehicle group, Bevacizumab decreased the leakage area significantly $(p=0.025$ and $p=0.040$ ). PRO-169 also decreased the leakage area significantly (blue arrows in Figure 3). The average percent

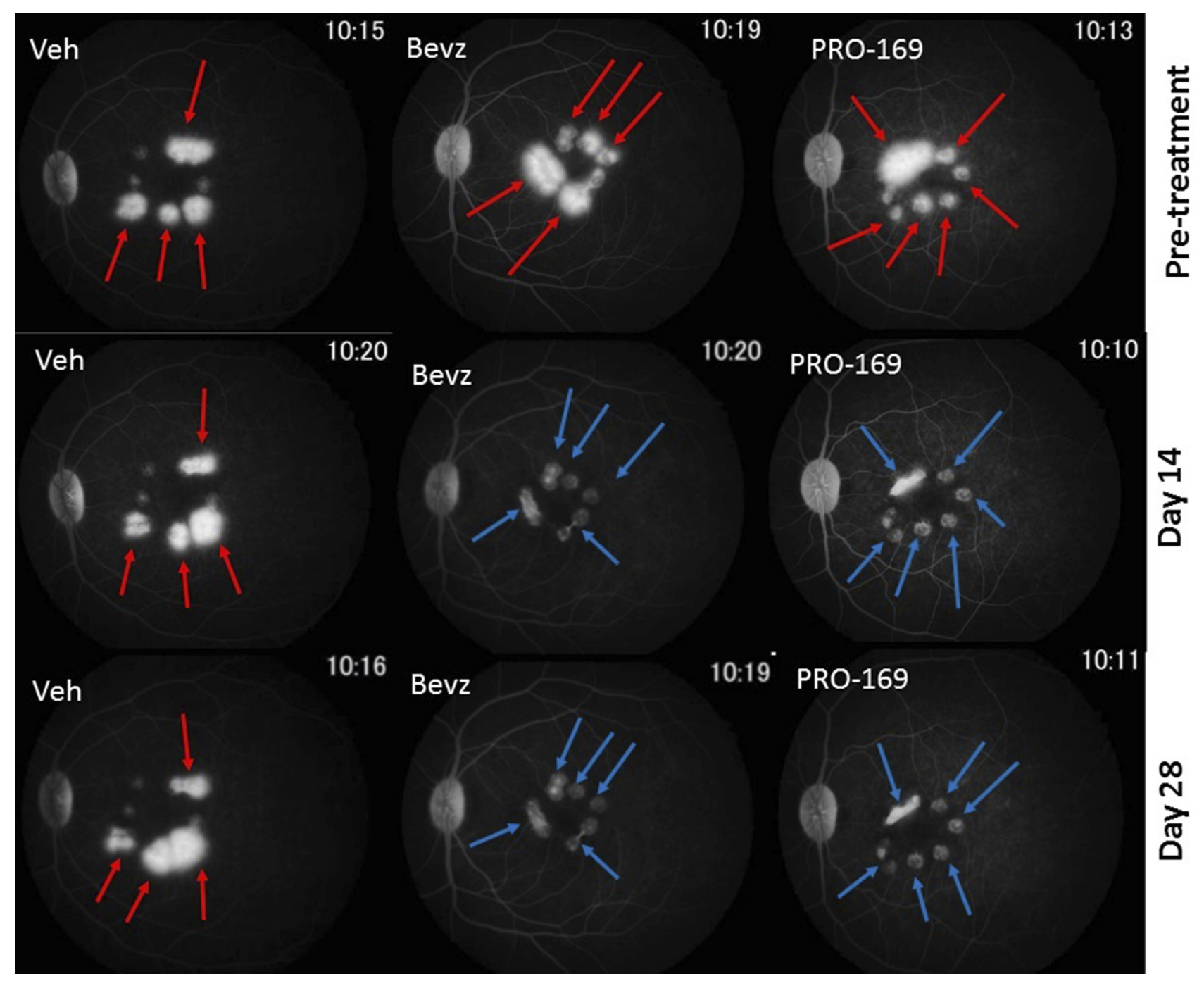

Figure 3 Bevacizumab and PRO-169 attenuate laser-induced CNV. Fluorescein fundus angiography (FFA) of vehicle (Veh), bevacizumab (Bevz) and PRO-I69 (IVT delivery of $1.25 \mathrm{mg} / \mathrm{eye}$, on post-operative day $2 \mathrm{I}$ ). Images provide representative examples of grade III and IV lesions identified by red arrows (pre-treatment phase), and the reduction of leakage area by blue arrows in Day 14 and Day 28 angiograms. 
Table 3 Change Of Leakage Area Of Grade III+IV Spots (\%)

\begin{tabular}{|l|l|l|l|l|}
\hline & $\begin{array}{l}\text { Vehicle, } \\
\text { n=7 Eyes }\end{array}$ & $\begin{array}{l}\text { Bevacizumab, } \\
\text { n=7 Eyes }\end{array}$ & $\begin{array}{l}\text { PRO-169, } \\
\text { n=7 Eyes }\end{array}$ & $\mathbf{p}$ \\
\hline Day 14 & $-27.6 \pm 35.3$ & $-75.3 \pm 49.4^{*}$ & $-82.0 \pm 19.3^{*}$ & 0.025 \\
Day 28 & $-36.1 \pm 34.4$ & $-78.0 \pm 42.6^{*}$ & $-81.4 \pm 21.0^{*}$ & 0.040 \\
\hline
\end{tabular}

Notes: Data are presented as \% mean \pm SD. One-way ANOVA, *compared with vehicle group.

change of leakage area was $-82.0 \pm 19.3 \%$ and -81.4 $\pm 21.0 \%$ at Day 14 and Day 28, respectively, see Table 3 . Compared with vehicle group, PRO-169 decreased the leakage area significantly $(p=0.032$ and $p=0.05$ ). No significant differences were observed between bevacizumab group and PRO-169 group at any time point ( $p=0.937$ and $p=0.980$, respectively).

\section{Amount Of Grade III And Grade IV Spots}

Fluorescein leakage spots were found in monkey's eyes 19 days after laser photocoagulation (pre-treatment Figure 2). The amount of grade III and IV spots was calculated as a sign of efficacy. In the eyes of vehicle group, the amount of grade III and IV spots did not change significantly compared with pre-treatment ( 31 vs 30 and 28 , respectively).

In the eyes of bevacizumab group, compared with vehicle, the amount of grade III and IV leakage spots reduced significantly at Day 14 (30 vs $\left.17, \chi_{(1)}^{2}=18.632, p=0.0001\right)$ and Day 28 ( 28 vs 17, $\left.\chi_{(1)}^{2}=13.209, p=0.0001\right)$. Meanwhile, in the eyes of PRO-169 group, the amount of grade III and IV leakage spots reduced significantly compared with vehicle at Day 14 (30 vs 21, $\left.\chi_{(1)}^{2}=9.908, p=0.003\right)$ and Day 28 (28 vs 21, $\chi_{(1)}^{2}=5.557, p=0.032$ ). No significant differences were observed between bevacizumab group and PRO-169 group at any time point $(p=0.218)$.

\section{Clinical Observations \\ Cage-Side Observations}

There were no significant abnormalities in behavior, autonomic activity, skin and hair, excretion and overall eye appearance before and after administration in all groups.

\section{Body Weight}

After the injection of vehicle or bevacizumab, the body weight of animals decreased at Day $14(-1.8 \pm 2.5 \%$ vs $-1.9 \pm 5.0 \%)$ and Day $28(-4.4 \pm 3.2 \%$ vs $-1.1 \pm 5.4 \%)$. However, the body weight of animals that receiving PRO-169 increased compared with vehicle on Day 28 $(-4.4 \pm 3.2 \%$ vs $2.3 \pm 1.5 \%), p=0.05$. No significant differences were observed in body weight between bevacizumab group and PRO-169 group at Day 14 and Day $28(p=0.587$ and $p=0.360$, respectively).

\section{Discussion}

The process of angiogenesis is multi-factorial and complex and VEGF is considered playing a critical in angiogenesis role. ${ }^{3}$ Ocular neovascularization provides an ideal in vivo system in which to investigate the mechanism that control angiogenesis. ${ }^{7}$ Abnormalities or defects in Brunch's membrane induce hypoxia, oxidative stress, and inflammation, which affect the balance of antiangiogenic factors in the direction of angiogenesis. ${ }^{23}$ Levels of angiogenic factors have shown to be related to the extent of CNV in vivo and antiangiogenic molecules that target VEGF are used in the management of AMD. ${ }^{8,11,23}$ AMD is a prevalent and debilitating disease with a large demand for treatments. New drugs are continuously being designed and the importance of cost-effectiveness to keep health care sustainable is growing. ${ }^{9}$ VEGF inhibitors have been shown to slow down visual loss, and improve vision in AMD patients. Bevacizumab is a mAb used off-label to treat neovascular AMD and other ocular diseases associated with macular edema and abnormal vessel growth. ${ }^{10,15}$ Bevacizumab is a cost-effective, safe treatment option for neovascular agerelated macular degeneration. ${ }^{9}$ In the current study, we designed a mAb PRO-169 structurally similar to bevacizumab but specifically for ophthalmic use. PRO-169 is a recombinant, humanized-anti-VEGF that having a target specificity like bevacizumab. ${ }^{9,14}$

Animal models of an ocular disease that is like the human condition would also be similar to humans in the efficacy, potency, and duration of action of drugs. These models are easier to validate for follow-on molecules in the same class of pharmacotherapy. ${ }^{24}$ However, bevacizumab and ranibizumab do not cross-react with mouse or rat VEGF; therefore, non-transgenic rodent models cannot be used. $^{10,16-19,25}$ PRO-169 is specific to human VEGF and does not bind murine VEGF. Its use for studies in mouse models is rather limited. ${ }^{20}$

The anatomic similarity between eyes of rhesus monkey and human provides theoretical advantages of NHP CNV models over other neovascularization models. ${ }^{21,22}$ Antibodies to VEGF have shown to inhibit neovascularization in an experimental model of laser-induced CNV in rhesus monkeys. The penetration of bevacizumab through the monkey retina after the intravitreal injection has been demostrated. The penetrating volume is sufficient to induce the therapeutic effects successfully in monkeys. ${ }^{26}$ 
We observed laser-induced CNVs in these monkeys' eyes are similar to previous reports in other NHPs..$^{1,3,5,12}$ Several reports have defined clinically relevant laserinduced CNV of grade III and IV lesions. However, graded scoring of fluorescein angiograms still requires a somewhat subjective assessment by examiners. ${ }^{1,26}$

Our findings demonstrated the inhibition effect of bevacizumab on laser-induced grade III-IV CNV was similar to previous studies. FFA was performed in all animals 19 days later to assess the severity and development of CNV following the laser treatment. There were no significant differences in the lesions diameter sizes or surface area in any eye of the vehicle or treatment groups before intravitreal injections. The change percentage of retinal thickness of the laser spot with the maximum retinal thickness was calculated as a sign of efficacy. The data from our study showed that the CNV induced by laser in vehicle group, which progressed during 14 and 28 days, was reduced after intravitreal injection of PRO-169. The average change percentage of retinal thickness was -131.6 $\pm 68.7 \%$ and $-131.5 \pm 63.8 \%$ on Day 14 and Day 28, respectively, without significant differences between bevacizumab and PRO-169 groups at any time point. IVT injection of PRO-169 can reduce the retinal thickness at CNVs for at least 28 days significantly. The effect is similar to ranibizumab from previous studies. ${ }^{12}$ Also, PRO-169 can decrease the average percent change of leakage area of grade III and IV spots without significant differences between bevacizumab and PRO-169. A single IVT injection of PRO-169 (1.25 mg per eye) decreased leakage throughout the follow-up period suggesting that the duration of action on vascular permeability with one injection of PRO-169 is at least 4 weeks. In agreement with previous studies, the intravitreal administration of the current clinical doses of bevacizumab and ranibizumab would result in the effective VEGF inhibition of relatively similar duration, $\sim 30$ days post-injection. ${ }^{12,19,27}$

A limitation of this study remains the low number of animal that can be ethically used. This limitation commonly results in studies with low statistical power. ${ }^{4}$ However, using a model with anatomic similarity to the human eye clearly compensates this limitation. We carried out this study using a sample of 21 primate eyes and encountered no adverse event in any eye. There were no significant abnormalities in behavior, skin and hair, excretion and in appearance before and after intravitreal injections in all groups. We believe that the current study provides sufficient basis that PRO-169 appears to be safe like bevacizumab in NHPs and that clinical studies may be undertaken.

In summary, after laser photocoagulation, the eyes enrolled in this study showed CNV related characteristics including increased retinal thickness, and fluorescein leakage at laser spots. Our data indicate that intravitreal injection of PRO-169 appears to be safe and can reduce the retinal thickness and fluorescein leakage for 14 and 28 days without toxic effect or adverse events. These findings suggested that PRO-169 can inhibit CNV in the same manner as bevacizumab (Avastin) for $\mathrm{CNV}^{4}$

In conclusion, PRO-169 treatment should be carefully evaluated in future clinical trials to confirm the safety and efficacy in the treatment of CNV. After photocoagulation, the eyes enrolled in this studio showed CNV related characteristics including increased retinal thickness, and fluorescein leakage at laser spots. PRO-169 (1.25 mg per eye) can reduce the retinal thickness and fluorescein leakage area after treatment for 14 and 28 days in this rhesus monkey model, without toxic effect or adverse events. These findings suggested that PRO-169 can inhibit CNV.

\section{Abbreviations}

AMD, age-related macular degeneration; CNV, choroidal neovascularization; FFA, fluorescein fundus angiography; FP, fundus photography; NHP, non-human primate; OCT, optical coherence tomography; VEGF, vascular endothelial growth factor.

\section{Acknowledgments}

We thank Sichuan Primed Shines for providing the experimental monkeys and conducting the monkey study and for their help during the experiment. This study was sponsored by Laboratorios Sophia, SA de CV (Zapopan, Jalisco, Mexico).

\section{Author Contributions}

All authors contributed to data analysis, drafting or revising the article, gave final approval of the version to be published, and agree to be accountable for all aspects of the work.

\section{Disclosure}

Olvera-Montaño O, Baiza-Duran L and Muñoz-Villegas P are employees of Laboratorios Sophia, SA de CV. Quintana-Hau JD and Quiñonez-Alvarado MG are employees of Centro de Investigación Sophia (CIS). The authors report no other conflicts of interest in this work. 


\section{References}

1. Goody RJ, Hu W, Shafiee A, et al. Optimization of laser-induced chroidal neovascularization in African green monkeys. Exp Eye Res. 2011;92:464-472. doi:10.1016/j.exer.2011.03.006

2. Krzystolik MG, Afshari MA, Adamis AP, et al. Prevention of experimental choroidal neovascularization with intravitreal anti-vascular endothelial growth factor antibody fragment. Arch Ophthalmol. 2012;120(3):338-346. doi:10.1001/archopht.120.3.338

3. Zhang M, Zhang J, Yan M, et al. Recombinant anti-vascular endothelial growth factor fusion protein efficiently suppresses choridal neovascularization in monkeys. Mol Vis. 2008;14:37-49.

4. Lichtlen P, Lam TT, Nork TM, et al. Relative contribution of VEGF and TNF- $\alpha$ in the cynomolgus laser-induced CNV model: comparing the efficacy of bevacizumab, adalimumab, and ESBA105. Invest Ophthalmol Vis Sc. 2010;51(9):4738-4745. doi:10.1167/iovs.09-4890

5. Lai K, Jin C, Tu S, et al. Intravitreal injection of 99Tc-MDP inhibits the development of laser-induced choroidal neovascularization in rhesus monkeys. Grafes Arch Clin Exp Ophthalmol. 2014;252:1049-1057. doi:10.1007/s00417-013-2559-1

6. Ding X, Patel M, Chan -C-C. Molecular pathology of age-related macular degeneration. Prog Retin Eye Res. 2009;28(1):1-18. doi:10.1016/j.preteyeres.2008.10.001

7. Miller JW, Adamis AP, Shima DT, et al. Vascular endothelial growth factor/vascular permeability factor is temporally and spatially correlated with ocular angiogenesis in a primate model. Am J Pathol. 1994;145(3):574-584.

8. Kaiser PK. Antivascular endothelial growth factor agents and their development: therapeutic implications in ocular diseases. $\mathrm{Am} J$ Ophthalmol. 2006;142(4):660-668. doi:10.1016/j.ajo.2006.05.061

9. Van Asten F, Michels CTJ, Hoyng CB, et al. The cost-effectiveness of bevacizumab and aflibercept for the treatment of age-related macular degeneration-a cost-effectiveness analysis from a societal perspective. PLoS One. 2018;13(5):e0197670. doi:10.1371/journal. pone. 0197670

10. Yu L, Wu X, Cheng Z, et al. Interaction between bevacizumab and murine VEGF-A: a reassessment. Invest Ophthalmol Vis Sci. 2008;49 (2):522-527. doi:10.1167/iovs.07-1175

11. Rodríguez EB, Farah ME, Maia M, et al. Therapeutic monoclonal antibodies in ophthalmology. Progs Retin Eye Res. 2009;28(2):117144. doi:10.1016/j.preteyeres.2008.11.005

12. Husain D, Kim I, Gauthier D, et al. Safety and efficacy of intravitreal injection of ranibizumab in combination with verteporfin PDT on experimental choroidal neovascularization in the monkey. Arch Ophthalmol. 2005;123:509-516. doi:10.1001/ archopht.123.4.509

13. CATT Research Group; Martin DF, Maguire MG, et al. Ranibizumab and bevacizumab for neovascular age-related macular degeneration. N Engl J Med. 2011;364(20):1897-1908.
14. Zhang Y, Han Q, Ru Y, et al. Anti-VEGF treatment for myopic choroid neovascularization: from molecular characterization to update on clinical application. Drug Des Devel Ther. 2015;9:314321.

15. Meyer CH, Holz FG. Preclinical aspects of anti-VEGF agents for the treatment of wet AMD: ranibizumab and bevacizumab. Eye. 2011;25 (6):661-672. doi:10.1038/eye.2011.66

16. Lu F, Adelman RA. Are intravitreal bevacizumab and ranibizumab effective in a rat model of choroideal neovascularization? Grafe's Arch Clin Exp Ophthalmol. 2009;247(2):171-177. doi:10.1007/ s00417-008-0936-y

17. Manzano RPA, Peyman GA, Khan P, et al. Inhibition of experimental corneal neovascularisation by bevacizumab (Avastin). $\mathrm{Br} \quad \mathrm{J}$ Ophthalmol. 2007;91:804-807. doi:10.1136/bjo.2006.107912

18. Akar EE, Öner V, Küçükerdönmez C, et al. Comparison of subconjunctivally injected bevacizumab, ranibizumab, and pegaptanib for inhibition of corneal neovascularization in a rat model. Int $J$ Ophthalmol. 2013;6(2):136-140. doi:10.3980/j.issn.2222-3959.2013.02.05

19. Pan CK, Durairaj C, Kompella UB, et al. Comparison of long-acting bevacizumab formulations in the treatment of choroidal neovascularization in rat model. J Ocul Pharmacol Ther. 2011;27(3):219-224. doi:10.1089/jop.2010.0158

20. Fuh G, Wu P, Liang WC, et al. Structure-function studies of two synthetic anti-vascular endothelial growth factor Fabs and comparison with Avastin Fab. J Biol Chem. 2006;281(10):6625-6631. doi:10.1074/jbc.M507783200

21. Lin $X$, Wang $Q, H e ~ M$. Repeated retinal photocoagulation in monkeys for the optimization of laser-induced choroidal neovascularization model. Exp Eyes Res. 2019;184:1-7. doi:10.1016/j.exer.2019.03.020

22. Mordenti J, Cuthbertson RA, Ferrara N, et al. Comparison of the intraocular tissue distribution, pharmacokinetics, and safety of 125 I-labeled full-length and fab antibodies in rhesus monkeys following intravitreal administration. Toxicol Pathol. 1999;27(5):536-544. doi: $10.1177 / 019262339902700507$

23. Seo S, Suh W. Antiangiogenic effect of dasatinib in murine models of oxygen-induced retinopathy and laser-induced choroidal neovascularization. Mol Vis. 2017;23:823-831.

24. Novack GD. Translating drugs from animals to humans: do we need to prove efficacy? Trans Vis Sci Tech. 2013;2(6):1. doi:10.1167/tvst.2.6.1

25. Miki K, Miki A, Matsuoka M, et al. Effects of intraocular ranibizumab and bevacizumab in transgenic mice expressing human vascular endothelial growth factor. Ophthalmology. 2009;116(9):1748-1754. doi:10.1016/j.ophtha.2009.05.020

26. Heiduschka P, Fietz H, Hofmeister S, et al. Penetration of bevacizumab through the retina after intravitreal injection in the monkey. Invest Ophthalmol Vis Sci. 2007;48(6):2814-2823. doi:10.1167/iovs.06-1171

27. Papadopoulos N, Martin J, Ruan Q, et al. Binging and neutralization of vascular endothelial growth factor (VEGF) and related ligands by VEGF Trap, ranibizumab and bevacizumab. Angiogenesis. 2012;15:171-185. doi:10.1007/s10456-011-9249-6

\section{Publish your work in this journal}

Drug Design, Development and Therapy is an international, peerreviewed open-access journal that spans the spectrum of drug design and development through to clinical applications. Clinical outcomes, patient safety, and programs for the development and effective, safe, and sustained use of medicines are a feature of the journal, which has also been accepted for indexing on PubMed Central. The manuscript management system is completely online and includes a very quick and fair peer-review system, which is all easy to use. Visit http://www. dovepress.com/testimonials.php to read real quotes from published authors. 